\title{
Genitourinary Syndrome of Menopause: Epidemiology, Physiopathology, Clinical Manifestation and Diagnostic
}

\author{
Ayane Cristine Alves Sarmento ${ }^{1 *}$, Ana Paula Ferreira Costa ${ }^{1}$, Pedro Vieira-Baptista ${ }^{2,3}$, \\ Paulo César Giraldo ${ }^{4}$, José Eleutério Jr. ${ }^{5}$ and Ana Katherine Gonçalves ${ }^{1,6}$ \\ ${ }^{1}$ Postgraduate Program in Health Sciences, Federal University of Rio Grande do Norte, Natal, Brazil, ${ }^{2}$ Lower Genital Tract \\ Unit, Centro Hospitalar de São João, Porto, Portugal, ${ }^{3}$ Hospital Lusíadas Porto, Porto, Portugal, ${ }^{4}$ Department of Obstetrics \\ and Gynecology, State University of Campinas, Campinas, Brazil, ${ }^{5}$ Department of Obstetrics and Gynecology, Federal \\ University of Ceará, Fortaleza, Brazil, ${ }^{6}$ Department of Obstetrics and Gynecology, Federal University of Rio Grande do Norte, \\ Natal, Brazil
}

OPEN ACCESS

Edited by:

Laura Buggio,

IRCCS Ca' Granda Foundation Maggiore Policlinico Hospital, Italy

Reviewed by:

Antonio Simone Laganà,

University of Insubria, Italy Andrea Giannini,

University of Pisa, Italy

*Correspondence: Ayane Cristine Alves Sarmento

ayane_cris@hotmail.com

Specialty section:

This article was submitted to

Gynecology,

a section of the journal

Frontiers in Reproductive Health

Received: 18 September 2021

Accepted: 21 October 2021

Published: 15 November 2021

Citation:

Sarmento ACA, Costa APF, Vieira-Baptista P, Giraldo PC, Eleutério

$J$ and Gonçalves AK (2021)

Genitourinary Syndrome of Menopause: Epidemiology,

Physiopathology, Clinical

Manifestation and Diagnostic. Front. Reprod. Health 3:779398. doi: 10.3389/frph.2021.779398
Genitourinary syndrome of menopause (GSM) is a term used to define a compilation of signs and symptoms arising from decreased estrogenic stimulation of the vulvovaginal and lower urinary tract. Among $27-84 \%$ of women in postmenopausal are affected for symptoms of GSM, and these can unquestionably impair health, sexual function, consequently the quality of life of these women. The main signs and symptoms of GSM include, among others, burning, irritation, vulvovaginal dryness, dyspareunia, urinary symptoms of urgency, dysuria, or recurrent urinary tract infection. The diagnosis can be made through anamnesis, questionnaires, physical exams, and, sometimes, complementary exams. Objective vaginal assessment is essential and can be complemented by using the Vaginal Health Index (VHI), Vaginal Maturation Index (VMI), or vaginal $\mathrm{pH}$ measurement. The acknowledgment of this condition by health professionals is crucial for its identification and proper management and exclusion of other conditions that make a differential diagnosis with it.

Keywords: menopause, genitourinary syndrome of menopause, vulvovaginal atrophy, epidemiology, diagnosis

\section{INTRODUCTION}

Menopause is a condition characterized by estrogen decline and results in numerous changes in the female organism. Vulvar and vaginal atrophy (VA) are among the most common and most bothersome ones $(1,2)$. The most prevalent symptom is vaginal dryness and affecting $60 \%$ of women in the postmenopausal period. In general, these symptoms lead to sexual dysfunction and consequently to reduced quality of life $(3,4)$.

In 2014, the International Society for the Study of Women's Sexual Health and the North American Menopause Society (NAMS) proposed the introduction of the designation of Genitourinary Syndrome of Menopause (GSM) to replace the term vulvovaginal atrophy (VVA), which has been using for a long time to describe the genitourinary changes of menopause. While several authors and societies use and endorse the concept $(5,6)$, the justification for its need, its definition, and even its use are still far from consensual. The GSM comprises numerous unspecific symptoms and signs. However, a minimum number of signs/symptoms required for the diagnosis of the syndrome was not defined. One of the major concerns is that given the lack of specificity of 
the vulvovaginal signs and symptoms, other conditions may easily be overlooked or inappropriate treatments may be provided $(7,8)$. Another critical question that needs to be taken into account is that VVA can occur also in hypoestrogenic states other than menopause: chemotherapy, pelvic radiation, progestin-only contraceptives, breastfeeding, or anti-estrogenic therapies. In addition, in specific situations, like breastfeeding or certain hormone treatments, it usually is unnecessary to treat women, as the VA resolves spontaneously when estrogen levels are restored (9).

The changes that provoke all the signs and symptoms are correlated with a decline in estrogens and other sex steroids. In general, these changes cause alterations in the clitoris, vaginal vestibule, labia majora and minora, urethra, and bladder. Generally, half of the menopausal and postmenopausal women presenting some signs and symptoms classic of GSM (10). Commonly, the diagnosis is established on the clinical assessment. The latter include anamnesis, assessment of the patient's symptoms, and gynecological examination with an appraisal of clinical signs, including evaluation of sexual function, decreased libido, and dyspareunia. Additionally, standardizer questionnaires and laboratory tests can be employed, such as vaginal $\mathrm{pH}$ and the Vaginal Maturation Index (VMI), the latter usually used only in research (11-13).

The increase in life expectancy that has taken place in recent years has meant that female populations live, on average, a third of their life in post-menopause. During this period, symptoms and diseases related to hypoestrogenism become increasingly crucial for women's health. For this reason, good knowledge about the GSM and trusting diagnosis can be valuable tools for the safety and efficacy treatment of these women.

\section{SEARCH STRATEGY}

We conducted a comprehensive search to the PubMed, Web of Science, Cochrane Library, and Scopus databases using the terms "(menopause OR post-menopause OR genitourinary syndrome of menopause) AND (epidemiology OR physiopathology OR clinical manifestation OR diagnostic OR treatment)" for studies published from inception until July 2021 yielding 5,562 manuscripts. Studies not specific about the themes and other narrative review articles were excluded. Finally, 53 studies were included in the review.

\section{EPIDEMIOLOGY}

Around the world, on average, women live longer than men. Are estimated that these women live more than 30 years following natural menopause, which commonly occurs between 48 and 52 years, in developed countries $(14,15)$. For this reason, the impact of potential conditions due to menopause-associated hormonal deficiency influences the healthy longevity of women $(16,17)$. With a prevalence ranging from 36 to $90 \%$, VVA affects many peri- and post-menopausal women. In pre-menopausal years, it can be found in 19\% of women aged 40-45 (18-21).
A study involving 2045 British women aged 55-85 years, demonstrated that $50 \%$ of these women present urogenital symptoms at some time of menopause. Some of these women were seriously affected; these symptoms are of extended duration and usually do not recede without appropriate treatment (22).

Another pan-European study that enrolled 3,000 women between the ages of 55-75 years and that evaluated the aspects of urogenital aging (UGA) observed that a total of $30 \%$ of women experiment from symptoms from urogenital atrophy, these, $60 \%$ using hormone therapy (HT) (23). Differences between the six involved European countries were reported, concerning women's perceptions on the use the HT, sexual relationships aspects, prevalence, and treatment of problems due to VVA. Effects of urogenital symptoms on sexual activity were also reported, including a decreased prevalence of sexual activity with increasing age. Over $70 \%$ of postmenopausal women were not sexually active, and more than $30 \%$ reported dyspareunia and vaginal dryness $(23,24)$.

Despite the latter result, it is important to refer that in other cohorts it was described that most postmenopausal women continue sexually active. In a study involving 94,000 postmenopausal women (50-79 years), 52\% related that who are sexually active with a partner in the last year (25). A review of the literature revealed that $22 \%$ of married women between 70 and 79 years still engaged in sexual intercourse $(26,27)$.

\section{PHYSIOPATHOLOGY}

The onset of menopause is defined as 12 months without menses. It is a predictable and expected fact in the climacteric. Therefore, endocrine events happen naturally, with its range of symptoms and signs similar to menarche, being also necessary as in this one, a phase of adaptation. There are several changes in ovarian structure and function, with a gradual decrease in estrogen production and a consequent increase in pituitary gonadotropins, characterizing a state of hypogonadotropic hypogonadism (7). During the menopause, the lack of steroid hormones makes the vaginal epithelium thinner due to a decrease in the number of layers of epithelial cells and degeneration of collagen and elastin fibers in the underlying connective tissue, resulting in less tissue elasticity and greater mucosal fragility. The latter changes can provoke burning, fissuring, dyspareunia, and post-coital bleeding. Additionally, the hypoestrogenism induces a decline in the vaginal epithelium glycogen level, which is the substrate for lactobacilli $(7,8)$.

In women undergoing hysterectomy, the onset of menopause occurs artificially, although the ovaries keep functioning. In situations of bilateral oophorectomy, menopause may be accompanied by the clinical manifestations of hypoestrogenism, occurring more frequently and more intensely than in menopause natural (5-8).

\section{CLINICAL MANIFESTATIONS}

The vaginal epithelium gets atrophic characteristics in postmenopausal women, presenting surfaces epithelial surfaces with 
TABLE 1 | Clinical manifestations of GSM.

\begin{tabular}{lll}
\hline Genital & Sexual & Urinary \\
\hline $\begin{array}{l}\text { Clinical manifestations } \\
\text { Vaginal dryness }\end{array}$ & $\begin{array}{l}\text { Sexual dyspareunia } \\
(21,24,32-36)\end{array}$ & Dysuria (41-44) \\
Irritation/burning/itching & $\begin{array}{l}\text { Reduced lubrication } \\
(37-40)\end{array}$ & Urgency (41-44) \\
$(21,24,32-36)$ & Post-coital bleeding & Incontinence \\
Leukorrhea & $(37-40)$ & $(41-44)$ \\
$(21,24,32-36)$ & Decreased orgasm and & Stress/urgency \\
Thinning/graying pubic & desire (37-40) & $(41-44)$ \\
hair (21, 24, 32-36) & Loss of libido (37-40) & Recurrent urinary \\
Vaginal/pelvic pain and & & tract infections \\
pressure (21, 24, 32-36) & & $(41-44)$ \\
& & Urethral prolapse \\
Vaginal vault prolapse & Dysorgasmia (37-40) & $(41-44)$ \\
$(21,24,32-36)$ & & Ischemia of vesical \\
Reduced lubrication & & trigone (41-44) \\
$(21,24,32-36)$ & &
\end{tabular}

Created by the authors.

features of keratinization and the absence of rugae, presenting numerous tiers of parabasal cells, and few intermediate and superficial cells $(28,29)$.

The GSM includes symptoms such as genital alterations of dryness, burning, and irritation, sexual changes, such as the absence of lubrication, discomfort, pain, impaired function until urinary symptoms (urgency, dysuria) $(8,30,31)$ (Table 1).

\section{Genital Symptoms}

The vaginal wall presents an abundance of estrogen receptors, especially in the mucosal epithelium, and to a lesser extent in the fibroblasts and the stromal smooth muscle. In the vagina are present two types of estrogen receptors ( $\alpha$ and $\beta$ ). The $\alpha$ receptors being present principally in the epithelium, the stroma, and the muscle fibers, while $\beta$ receptors predominate in the blood vessel endothelium. Besides, androgen receptors also are present in the epithelium and the stroma of the vagina (32-34).

Under these circumstances, the vaginal epithelium becomes thinner, inflamed, often with erosions and bleeding $(24,35,36)$. Clinical signs associated with histological changes at the vaginal include burning, dryness, loss of elasticity, petechiae, ulceration, inflammation, discharge fibrosis, and vaginal obliteration. The most common vulvar signs include reduction in tissue thickness (loss of fat), labia agglutination, and loss of pubic hair $(2,21,45)$ (Figures 1A,B).

\section{Sexual Symptoms}

Coital activity can be uncomfortable in the postmenopausal period because of poor lubrication. In addition, there is a loss of rugae, occasionally stenosis, and the prepuce of the clitoris atrophies $(24,46)$.

Most common symptoms include vaginal dryness (78\%) and superficial dyspareunia (76\%). Other symptoms include burning, the pain increased susceptibility to physical and chemical

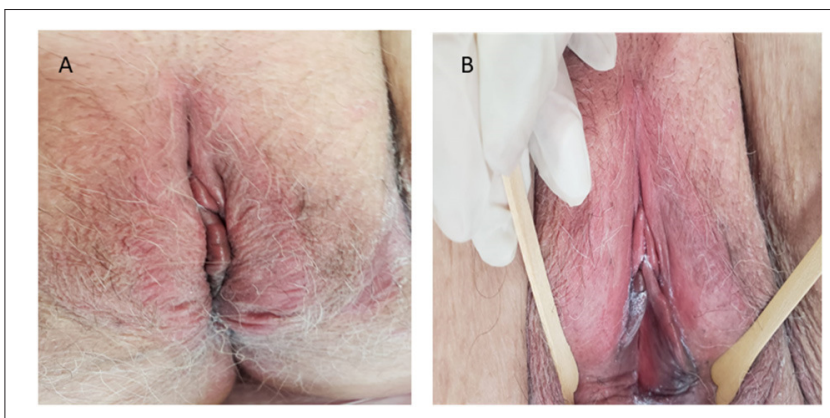

FIGURE 1 | Vulvar during menopause (Authors' personal archive).

irritation. All of these symptoms affect postmenopausal women's sexuality and relationships $(37,38)$.

A critical study performed in Spain showed that VVA symptoms significantly affect women's capacity to achieve pleasurable relations (74\%) and spontaneity (70\%) (39). Another study performed in 2014 demonstrated that most women were concerned discomfort could interfere with your relationship have long-term (40). One crucial point that could aggravate the sexual symptoms is that most women do not seek assistance, considering it's a natural part of aging (24).

\section{Urinary Symptoms}

Due to the bladder trigone and the urethra contain countless estrogen receptors decline in muscle mass and strength when serum estrogen declines provoke the postmenopausal women not to use hormonal therapy to have higher muscle damage $(34,35)$.

Urogenital symptoms include frequency, nocturia, urgency, and incontinence. Cystitis associated with the estrogen deficit, may not always be acknowledged. The impact of hypoestrogenism is especially relevant in the urethral epithelium, leading to decline sensitivity of urethral smooth muscle, decreased collagen, and loss of urethral wrinkles. The sum of all these changes generally is referred to as the urethral syndrome $(41,42)$.

Most women (70\%) associate the beginning of urinary incontinence with their last menstrual period. Twenty percent refer to grave urgency, and approximately 50\% complain of stress incontinence. Urge incontinence is more frequent, and it increases over the years of estrogen deficiency. Shear forces, limited mobility, tissue atrophy, slower dissipation, excess skin hydration, lower tissue regeneration capacity are factors that increase the chances of morbidity development from incontinence dermatitis in postmenopausal women $(24,43,44)$.

\section{DIAGNOSTIC}

The clinical evaluation of climacteric women should focus on current and past health status and involve a multidisciplinary team. Attention needs encompass health promotion, disease prevention, symptom care clinical and possible difficulties of this phase. Systemic pathologies may occur concomitantly, reverberating in complaints such as joint or muscle pain, gradual 
weight gain, depression, or even symptoms of undiagnosed hypothyroidism, simulated by coincidence in the ovarian hormonal transition. The VVA diagnosis is based on clinical assessment (anamnesis) and gynecological examination. Besides the use of standardized questionnaires and laboratory exams, such as the vaginal $\mathrm{pH}$ and the VMI. Questions about sexual function (including decreased libido), and dyspareunia should be including in the anamnesis $(2,8,12,20,21,31,46)$.

\section{Vaginal pH}

The $\mathrm{pH}$ considered normal during the fertile periods of women is among the values of 4.0 and 4.7. The latter occurs due to the presence of lactic acid, which provokes acidifies the medium. In most studies, the vaginal $\mathrm{pH}$, frequently, is measured using a $\mathrm{pH}$ indicator strip on the sidewall of the vagina. For this reason, the measurement of vaginal $\mathrm{pH}$ is considered useful, effective, and cheap $(12,24,46-48)$. However, it is considered unspecific since other factors, such as infections or recent intercourse, can influence the $\mathrm{pH}$ vaginal.

\section{Vaginal Maturation Index (VMI)}

The VMI is used to demonstrate the degree of tissue maturation. This measurement is realized for a count of the percentage of superficial, intermediate, and parabasal cells. The vaginal cytology enables a mean index of these three types of cells calculated from at least 100 smear cells. Because the atrophic vaginal tissue can't realize the process of maturation from parabasal and intermediate cells to superficial cells. The percentage of superficial cells correspondents $<5 \%$, it indicates an atrophic smear $(2,8,13,31,48,49)$. In clinical practice, the use of VMI is unneeded, but it is commonly used in research.

\section{Vaginal Health Index (VHI)}

One of the most common clinical tools used for the evaluation of VVA symptoms is the VHI. This tool evaluates five parameters (vaginal elasticity, vaginal secretions, $\mathrm{pH}$, epithelial mucous membrane, vaginal hydration). The degree of atrophy of the vagina is defined by the final score obtained after the evaluation of each parameter. The final score can vary between 5 and 25 , with a cut-off $<15$ meaning that there is a VA vagina $(2,12,31,50)$.

\section{TREATMENT}

The main objective of treating GSM is to alleviate symptoms provided by that period. First-line therapies for less severe symptoms include non-hormone vulvar and vaginal lubricants and long-acting vaginal moisturizers used regularly. Although not supported by clinical trials, regular, gentle vaginal stretching exercises may reduce GSM symptoms. Prescription therapies include low-dose vaginal estrogens, vaginal DHEA inserts, and oral ospemifene. For women with moderate to severe dyspareunia associated with GSM with concurrent VMS, transdermal and oral HT are effective options. Pelvic floor muscle training significantly reduces VVA in postmenopausal women. The use of energy-based also has been proposed, and devices are thought to improve vaginal health by causing microtrauma, which induces collagen formation, angiogenesis, and epithelial thickening. The fractional $\mathrm{CO}_{2}$ laser has demonstrated safety and efficacy for improving GSM symptoms of vaginal dryness and dyspareunia $(6,8,32,49)$.

Another new proposal includes the use of Visnadine, an active ingredient of the fruit of Ammi visnaga. The latter demonstrates potent vasodilatory activity and has ameliorated female sexual arousal disorder (51). Associated a Visnadine, prenylflavonoids, and phytoestrogens have been considered due to their potential role as estrogen receptor alpha selective agonist and may counteract the effects of postmenopausal estrogen loss. Recently, a vaginal cream containing bovine colostrum has effectively relieved vaginal dryness and other VVA symptoms in postmenopausal women after 8 weeks of treatment (52).

Research realized using the Aloe Vera extract showed a significant improvement in both the VHIS and FSFI, which can be considered direct and indirect signs of increased vaginal epithelialization. All the most significant vaginal health parameters increased indirectly increased female sexual functions too. Finally, the evidence reported that Aloe Vera extracts have substantial antioxidant, antibacterial, antiinflammatory, and wound-healing properties. Future research is necessary (53).

\section{CONCLUSIONS}

GSM is a new term for a condition more renowned as atrophic vaginitis. It is characterized by a hypoestrogenic condition with external genital, urological, and sexual implications that affect postmenopausal women. Knowledge of the concept of GSM and its impact on the quality of life is of paramount importance since it affects millions of women worldwide. The most up-to-date literature about clinical manifestations, pathophysiology, etiology, evaluation and management of GSM is comprehensively reviewed. Early detection and individually tailored pharmacologic or non-pharmacologic. The treatment is paramount for improving quality of life and preventing exacerbation of symptoms in women with this condition. Knowledge of the concept of GSM and its impact on the quality of life is of paramount importance since it affects millions of women worldwide. The GSM is still an under-diagnosed and consequently under-treated condition since the postmenopausal woman does not seek help at the onset of symptoms, use treatments on their own to avoid medical consultation, or do not seek treatment because they believe that all the changes and symptoms are a normal aging process. Due to the latter gaps that still need to be clarified, further and clarifying on this topic are necessary.

\section{AUTHOR CONTRIBUTIONS}

ACAS and AKG conceived and designed the study. ACAS, $\mathrm{AKG}, \mathrm{APFC}$, and PV-B drafted and revised the article where appropriate. ACAS and APFC prepared the Table. AKG, PV-B, JE, and PCG carried out the final revision of the manuscript. All authors contributed to the article and approved the submitted version. 


\section{REFERENCES}

1. Palacios S, Castelo-Branco C, Currie H, Mijatovic V, Nappi RE, Simon J, et al. Update on management of genitourinary syndrome Menopause: a practical guide. Maturitas. (2015) 82:308-13. doi: 10.1016/j.maturitas.2015.07.020

2. Palacios S. Assessing symptomatic vulvar, vaginal, and lower urinary tract atrophy. Climacteric. (2019) 22:348351. doi: 10.1080/13697137.2019.1600499

3. Castelo-Branco C, Cancelo MJ, Villero J, Nohales F, Julia' MD. Management of post-menopausal vaginal atrophy and atrophic vaginitis. Maturitas. (2005) 52:46-52. doi: 10.1016/j.maturitas.2005.06.014

4. Nappi RE, Palacios S. Impact of vulvovaginal atrophy on sexual health and quality of life at postmenopause. Climacteric. (2014) 17:3-9. doi: 10.3109/13697137.2013.871696

5. Portman DJ, Gass ML. Vulvovaginal atrophy terminology consensus conference panel. Genitourinary syndrome of menopause: new terminology for vulvovaginal atrophy from the International Society for the Study of Women's Sexual Health and the North American Menopause Society. Menopause. (2014) 21:1063-8. doi: 10.1097/GME.0000000000000329

6. NAMS. The 2020 genitourinary syndrome of menopause position statement of The North American Menopause Society. Menopause. (2020) 27:976992. doi: 10.1097/GME.0000000000001609

7. Pérez-López FR, Vieira-Baptista P, Phillips N, Sacher BC, Susana CAV, Fialho SCAV, et al. Clinical manifestations and evaluation of postmenopausal vulvovaginal atrophy. Gynecol Endocrinol. (2021) 26:1-6. doi: 10.1080/09513590.2021.1931100

8. Sarmento ACA, Fernandes FS, Costa APF, Medeiros KS, Crispim JC, Gonçalves AK. Microablative fractional radiofrequency for the genitourinary syndrome of menopause: protocol of randomised controlled trial. BMJ Open. (2021) 11:e046372. doi: 10.1136/bmjopen-2020-046372

9. Vieira-Baptista P, Claudia Marchitelli C, Haefner HK. Deconstructing the genitourinary syndrome of menopause. Int Urogynecol J. (2017) 28:6759. doi: 10.1007/s00192-017-3295-6

10. Shifren JL. Genitourinary syndrome of menopause. Clin Obstet Gynecol. (2018) 61:508-16. doi: 10.1097/GRF.0000000000000380

11. Jannini EA, Nappi RE. Couplepause: a new paradigm in treating sexual dysfunction during menopause and andropause. Sex Med Rev. (2018) 6:38495. doi: 10.1016/j.sxmr.2017.11.002

12. Bachmann G. Urogenital ageing: an old problem newly recognized. Maturitas. (1995) 22:S1-5. doi: 10.1016/0378-5122(95)00956-6

13. Hess R, Austin RM, Dillon S, Chang CC, Ness RB. Vaginal maturation index self-sample collection in mid-life women: acceptability and correlation with physician-collected samples. Menopause. (2008) 15:726-9. doi: 10.1097/gme.0b013e31816c5541

14. GBD 2017 Mortality Collaborators. Global, regional, and national age-se specific mortality and life expectancy, 1950-2017: a systematic analysis for the Global Burden of Disease Study 2017. Lancet. (2018) 392:1684735. doi: 10.1016/S0140-6736(18)31891-9

15. Palacios S, Henderson VW, Siseles N, Tan D, Villaseca P. Age of menopause and impact of climacteric symptoms by geographical region. Climacteric. (2010) 13:419-28. doi: 10.3109/13697137.2010.507886

16. Monteleone P, Mascagni G, Giannini A, Genazzani AR, Simoncini T. Symptoms of menopause-global prevalence, physiology and implications. Nat Rev Endocrinol. (2018) 14:199-215. doi: 10.1038/nrendo.2017.180

17. Davis SR, Lambrinoudaki I, Lumsden M, Mishra GD, Pal L, Rees M, et al. Menopause. Nat Rev Dis Primers. (2015) 1:15004. doi: 10.1038/nrdp.2015.4

18. Nappi RE, Kokot-Kierepa M. Women's voices in the menopause: results from an international survey or vaginal atrophy. Maturitas. (2010) 67:2338. doi: 10.1016/j.maturitas.2010.08.001

19. Kingsber SA, Krychman M, Graham S, Bernick B, Mirkin S. The women's EMPOWER survey: identifying women's perceptions on vulvar and vaginal atrophy and its treatment. J Sex Med. (2017) 14:41324. doi: 10.1016/j.jsxm.2017.01.010

20. Nappi RE, Seracchioli R, Salvatore S, Cagnacci A, Di Paolantonio T, Busacca M. Impact of vulvovaginal atrophy of menopause: prevalence and symptoms in Italian women according to the EVES study. Gynecol Endocrinol. (2019) 35:453-9. doi: 10.1080/09513590.2018.1563883
21. Alvisi S, Gava G, Orsili I, Giacomelli G, Baldassarre M, Seracchioli R et al. Review: vaginal health in menopausal women. Medicina. (2019) 55:615. doi: 10.3390/medicina55100615

22. Barlow DH, Cardozo LD, Francis RM, et al. Urogenital ageing and its effect on sexual health in older British women. Br J Obstet Gynaecol. (1997) 104:8791. doi: 10.1111/j.1471-0528.1997.tb10655.x

23. Barlow DH, Samsioe G, van Geelen JM. A study of European women's experience of the problems of urogenital ageing and its management. Maturitas. (1997) 27:239-47. doi: 10.1016/S0378-5122(97)00047-9

24. Calleja-Agiusand J, Brincat MP. The urogenital system and the menopause. Climacteric. (2015) 18:18-22. doi: 10.3109/13697137.2015.1078206

25. Call-Hosenfeld JS, Jaramillo SA, Legault C, et al. Correlates of sexual satisfaction among sexually active postmenopausal women in the Women's Health Initiative Observational Study. J Gen Intern Med. (2008) 23:20009. doi: 10.1007/s11606-008-0820-9

26. Schneidewind-Skibbe A, Hayes RD, Koochaki PE, Meyer J, Dennerstein L. The frequency of sexual intercourse reported by women: a review of communitybased studies and factors limiting their conclusions. J Sex Med. (2008) 5:30135. doi: 10.1111/j.1743-6109.2007.00685.x

27. Portman DJ, Margery LS, Gass MD. Genitourinary syndrome of menopause: new terminology for vulvovaginal atrophy from the International Society for the Study of Women's Sexual Health and The North American Menopause Society. J Sex Med. (2014) 11:2865-72. doi: 10.1111/jsm.12686

28. Cagnacci A, Xholli A, Sclauzero M, Venier M, Palma F, Gambacciani M. Vaginal atrophy across the menopausal age: results from the ANGEL study. Climacteric. (2019) 22:85-9. doi: 10.1080/13697137.2018.1529748

29. Adabi K, Golshahi F, Niroomansh S, Razzaghi Z, Ghaemi M. Effect of the fractional $\mathrm{CO}_{2}$ laser on the quality of life, general health, and genitourinary symptoms in postmenopausal women with vaginal atrophy: a prospective cohort. J Lasers Med Sci. (2020) 11:65-69. doi: 10.15171/jlms.2020.11

30. Politano CA, Costa-Paiva L, Aguiar LB, et al. Fractional $\mathrm{CO}_{2}$ laser versus promestriene and lubricant in genitourinary syndrome of menopause. Menopause. (2019) 26:833-840. doi: 10.1097/GME.0000000000001333

31. Sarmento ACA, Lírio JF, Medeiros KS, Camila Marconi C, Costa APF, Crispim JC, et al. Physical methods for the treatment of genitourinary syndrome of menopause: a systematic review. Int J Gynecol Obstet. (2021) 00:1-20. doi: 10.1002/ijgo.13561

32. Perez-Lopez FR, Phillipsb N, Vieira-Baptistac P, Cohen-Sacherf B, Fialho SCAV, Stockdale CK. Management of postmenopausal vulvovaginal atrophy: recommendations of the International Society for the Study of Vulvovaginal Disease. Gynecol Endocrinol. (2021) 37:746-52. doi: 10.1080/09513590.2021.1943346

33. Martin-Alguacil N, Pfaff DW, Kow LM, Schober JM. Oestrogen receptors and their relation to neural receptive tissue of the labia minora. BJU Int. (2008) 101:1401-6. doi: 10.1111/j.1464-410X.2008.07626.x

34. Sawczuk B, GołeRbiewska M, Mazurek A, Chyczewski L. Immunohistochemical evaluation of oestrogen receptors $\mathrm{a}$ and $\mathrm{b}$ in epithelium of the vaginal mucous membrane in women after oestrogen therapy. Prz Menopauzalny. (2017) 16:12-8. doi: 10.5114/pm.2017.66178

35. Baldassarre M, Perrone AM, Giannone FA, Armillotta F, Battaglia C, Costantino A, et al. Androgen receptor expression in the human vagina under different physiological and treatment conditions. Int J Impot Res. (2013) 25:7-11. doi: 10.1038/ijir.2012.25

36. Brotman RM, Shardell MD, Gajer P, Fadrosh D, Chang K, Silver MI, et al. Association between the vaginal microbiota, menopause status, and signs of vulvovaginal atrophy. Menopause. (2018) 25:132130. doi: $10.1097 /$ GME. 0000000000001236

37. Sarmento AC, Fernandes FS, Marconi C, Giraldo CP, Janaina C, Crispim JC, et al. Impact of microablative fractional radiofrequency on the vaginal health, microbiota, and cellularity of postmenopausal women. CLINICS. (2020) 75:e1750. doi: 10.6061/clinics/2020/1750

38. Tadir Y, Iglesia C, Alexiades M, Davila GW, Guerette N, Gaspar A. Energy-based treatment for gynecologic conditions including genitourinary syndrome of menopause: consensus, controversies, recommendations, and FDA clearance. Lasers Surg Med. (2019)15:315-317. doi: 10.1002/lsm.23031

39. Palacios S, Cancelo MJ, Castelo Branco C, Llaneza P, Molero F, Borrego RS. Vulvar and vaginal atrophy as viewed by the Spanish REVIVE participants: 
symptoms, management and treatment perceptions. Climacteric. (2017) 20:55-61. doi: 10.1080/13697137.2016.1262840

40. Kingsberg SA, Wysocki S, Magnus L, Krychman ML. Vulvar and vaginal atrophy in postmenopausal women: findings from the REVIVE (Real Women's VIews of Treatment Options for Menopausal Vaginal ChangEs) survey. J Sex Med. (2013) 10:1790-9. doi: 10.1111/jsm.12190

41. Schiavi MC, Pinto A, Sciuga V, Faiano P, Di Tucci C, D'oria O, et al. Prevention of recurrent lower urinary tract infections in postmenopausal women with genitourinary syndrome: outcome after 6 months of treatment with ospemifene. Gynecol Endocrinol. (2017). doi: 10.1080/09513590.2017.1370645

42. Perrotta C, Aznar M, Mejia R, Albert X, Ng CW. Oestrogens for preventing recurrent urinary tract infection in postmenopausal women. Cochrane Database Syst Rev. (2008) 2:CD005131. doi: 10.1002/14651858.CD005131.pub2

43. Robinson D, Cardozo LD. The role of estrogens in female lower urinary tract dysfunction. Urology. (2003) 62:45-51. doi: 10.1016/S0090-4295(03)00676-9

44. Cardozo L, Robinson D. Special considerations in premenopausal and postmenopausal women with symptoms of overactive bladder. Urology. (2002) 60:64-71. doi: 10.1016/S0090-4295(02)01799-5

45. Klebanoff SJ, Hillier SL, Eschenbach DA, Waltersdorph AM. Control of the microbial flora of the vagina by $\mathrm{H}_{2} \mathrm{O}_{2}$-generating lactobacilli. J Infect Dis. (1991) 164:94-100. doi: 10.1093/infdis/164.1.94

46. Milsom I, Arvidsson L, Ekelund P, Molander U, Eriksson O. Factors influencing vaginal cytology, $\mathrm{pH}$ and bacterial flora in elderly women. Acta Obstet Gynecol Scand. (1993) 72:286-91. doi: 10.3109/00016349309068039

47. Angelou K, Grigoriadis T, Diakosavvas M, Zacharakis D, Athanasiou S. The genitourinary syndrome of menopause: an overview of the recent data. Cureus. (2020) 12:e7586. doi: 10.7759/cureus.7586

48. Robinson D, Cardozo L. Urinary Incontinence. In: Edmonds DK, editor. Dewhurst's Textbook of Obstetrics and Gynaecology. 7th ed. Oxford: Blackwell Publishing (2007). p. 504-59. doi: 10.1002/9780470753354.ch49

49. NAMS. Management of symptomatic vulvovaginal atrophy: 2013 position statement of The North American Menopause Society. Menopause. (2013) 20:888-902. doi: 10.1097/GME.0b013e3182a122c2

50. McEndree B. Clinical application of the vaginal maturation index. Nurse Pract. (1999) 24:48, 51-2, 55-6. doi: 10.1097/00006205-199909000-00004
51. Caruso S, Mauro D, Cariola M, Fava V, Rapisarda, A. Cianci. Randomized crossover study investigating daily versus on-demand vulvar Visnadine spray in women affected by female sexual arousal disorder, Gynecol. Endocrinol. (2017). doi: 10.1080/09513590.2017.13 54366

52. Laganà AS, Vitale, SG, Stojanovska L, Lambrinoudaki I, Apostolopoulos V, Chiofalo B, et al. Preliminary results of a single-arm pilot study to assess the safety and efficacy of visnadine, prenylflavonoids and bovine colostrum in postmenopausal sexually active women affected by vulvovaginal atrophy. Maturitas. (2018) 109:78-80. doi: 10.1016/j.maturitas.2017. 12.015

53. Laganà AS, Chiofalo $B$, Granese $R$, Palmar V, Triolo O. Effects of titanium dioxide microcrystals with covalently bonded silver ions and Aloe Vera extract (TIAGIN $®$ ) on the Vaginal Health Index Score (VHIS) and Female Sexual Function Index (FSFI) in patients with vaginal deepithelialization: a prospective, single-center cohort analysis. Ital J Gynecol Obstet March. (2017) 29:07-11.

Conflict of Interest: The authors declare that the research was conducted in the absence of any commercial or financial relationships that could be construed as a potential conflict of interest.

Publisher's Note: All claims expressed in this article are solely those of the authors and do not necessarily represent those of their affiliated organizations, or those of the publisher, the editors and the reviewers. Any product that may be evaluated in this article, or claim that may be made by its manufacturer, is not guaranteed or endorsed by the publisher.

Copyright (c) 2021 Sarmento, Costa, Vieira-Baptista, Giraldo, Eleutério and Gonçalves. This is an open-access article distributed under the terms of the Creative Commons Attribution License (CC BY). The use, distribution or reproduction in other forums is permitted, provided the original author(s) and the copyright owner(s) are credited and that the original publication in this journal is cited, in accordance with accepted academic practice. No use, distribution or reproduction is permitted which does not comply with these terms. 